\title{
Deep renal ectopia causing sciatic mononeuropathy
}

\author{
Reza Assadsangabi • Mehrdad Hajmomenian • \\ Seyed Ali Nabavizadeh • Arastoo Vossough
}

Received: 16 April 2014 / Accepted: 21 May 2014 / Published online: 6 June 2014

(C) Springer-Verlag Berlin Heidelberg 2014

\begin{abstract}
Introduction Sciatic mononeuropathy resulting from compression by an adjacent pelvic ectopic kidney has not been reported in the literature. Here, the authors present a case of a patient presenting with unilateral lower extremity paresthesia correlating to the sciatic nerve distribution.

Results MRI examination demonstrated lumbosacral plexus compression by a very unusual deeply situated renal ectopia. Physicians should consider other rare causes of mechanical lumbosacral plexus compression in patients with sciatic symptomatology.
\end{abstract}

Keywords Renal ectopia $\cdot$ Sciatica

\section{Introduction}

The incidence of a pelvic kidney is estimated between 1 in 2,200 and 1 in 3,000 [1]. Given the rarity of a pelvic kidney, it is often an incidental finding on imaging studies or an unsuspected finding in patients presenting with vague or atypical symptoms. Its proximity to various organs is responsible for its different presentations. Ectopic kidneys are typically located in the upper false pelvis and lower abdomen. There is no report in the literature linking a primary ectopic kidney and neuropathy; however, mononeuropathy mostly of the femoral

R. Assadsangabi · M. Hajmomenian · S. A. Nabavizadeh •

A. Vossough $(\bowtie)$

Radiology Department, The Children's Hospital of Philadelphia,

34th St and Civic Center Blvd, Philadelphia, PA, USA

e-mail: vossough@email.chop.edu

R. Assadsangabi

e-mail: assadsangr@email.chop.edu

A. Vossough

University of Pennsylvania, Philadelphia, PA, USA nerves as a complication of renal transplantation into the pelvis has been described [2]. Sciatic nerve involvement after renal transplant is exceedingly rare, and we found just one case report in the literature [3]. Here, we present a case of rare, deeply situated ectopic kidney in the true pelvis, resulting in sacral plexus compression and sciatic mononeuropathy.

\section{Case report}

A 16-year-old girl presented with paresthesia and numbness in the right big toe extending to the ball of the foot. This was more noticeable when she was sitting with her legs crossed. Symptoms exacerbated when she stood on her feet for several hours and spread to the medial side of her ankle. She denied any low back pain or any other radicular symptoms in the legs. There were no bladder difficulties or muscle weakness. The patient had a positive family history of multiple sclerosis.

There was no history of developmental abnormalities. The spine had a normal curvature with no external abnormalities. On examination, impaired pain and temperature sensation of the right great toe on all surfaces extending to the ball of the foot was demonstrated. Distal position and vibration sensations were decreased. Straight leg raising test was $90^{\circ}$ on both sides. There was a normal gait and cerebellar exam. There was no Tinel's sign over the tarsal tunnel or the fibular head region. She had full strength in all muscle groups of the lower extremities with no atrophy or fasciculations. Plantar reflexes were reactive. Routine blood and metabolic profile including glucose level was normal. Electrodiagnostic studies were unrevealing.

Given the history, physical exam, and family history of multiple sclerosis, MRI exams of the head and spine were performed. Brain MRI was normal. Spinal MRI was also normal for vertebra, disk, spinal canal, or spinal cord pathology. However, a very deep right-sided pelvic kidney was 
noted, which was situated in the right true pelvis in a much lower position than what is typical for ectopic kidneys. It was in close proximity to the right lumbosacral plexus, and it abutted and impinged the right L5 and S1 nerves as they coursed along the lumbosacral plexus to join in forming the sciatic nerve (Fig. 1). The upper pole of the ectopic kidney was situated anterior to the right aspect of the sacral promontory in a paramedian position, extending inferiorly to the level of the sciatic notch. In this small room between the kidney and sacrum, the lumbosacral plexus traversed to form the sciatic nerve. Some of the fat planes between the kidney and sacral plexus trunks were obliterated, with the kidney touching and impinging upon the traversing L5 and S1 trunks (Fig. 1).

The patient was treated with Gabapentin and the symptoms resolved for a period of time; however, it did recur, especially after prolonged standing. Increased dosage helped in stabilization of the patient's lower extremity symptoms. No surgery was performed given the nature of the symptoms and medical symptom control.

\section{Discussion}

Renal ectopia results from failure of cephalic migration of the embryonic kidneys to their normal retroperitoneal location.
The incidence of renal ectopia is reported between 1 in 2,200 and 1 in 3,000 [1]. However, a study screening 132,686 school children (6 to 15 years of age) found a lower incidence of 1 in 5000 children [4]. Ectopic kidneys are typically located in the upper false pelvis and lower abdomen. Renal ectopia situated in the true pelvis is exceedingly rare and can pose diagnostic difficulties [5].

Kidneys as the related cause of neuropathy are reported in post renal transplantation cases [2, 3]. The mechanisms of posttransplant neuropathy are diverse and mainly depend on the nerve involved as well as type of procedure utilized. This complication is mostly described for the femoral nerve, as the result of surgical trauma, compression, ischemia, entrapment, or drugrelated neurotoxicity $[2,3,6,7]$. Sciatic nerve involvement after renal transplant is exceedingly rare as the nerve trunk is typically beyond the surgical field. There is only one case report in the literature indicating sciatic neuropathy after renal transplantation [3]. This report hypothesized that local ischemia precipitated by surgery may have been the cause.

Sciatic mononeuropathy has not been previously reported as result of compression by an adjacent ectopic kidney as depicted in this case. Given the very rare position of the ectopic kidney in this case, mechanical sciatic nerve compression may be precipitated by the
Fig. 1 a Sagittal T1-weighted image shows a very deeply situated pelvic kidney (curved arrow) in the true pelvis between the sacrum and bladder. The L5 trunk of the sacral plexus is coursing inferiorly and being impinged between the ectopic kidney and sacrum (short arrow). The superior gluteal artery follows a close course (arrowhead). The traversing S1 root is also seen (long arrow). b Axial T2-weighted image shows L5 (short arrow) and S1 (long arrow) trunks as they course between the pelvic kidney and sacrum. The adjacent superior gluteal artery (arrowhead) is also seen. c, d Post contrast axial T1weighted images demonstrate the course of sacral trunk at and below the level of sacral promontory along with the superior gluteal artery (arrowhead). Note obliteration of the fat planes between the kidney and the nerves. The sacral plexus is impinged upon by adjacent ectopic kidney, including the L5 (short arrow) and S1 (long arrow) trunks
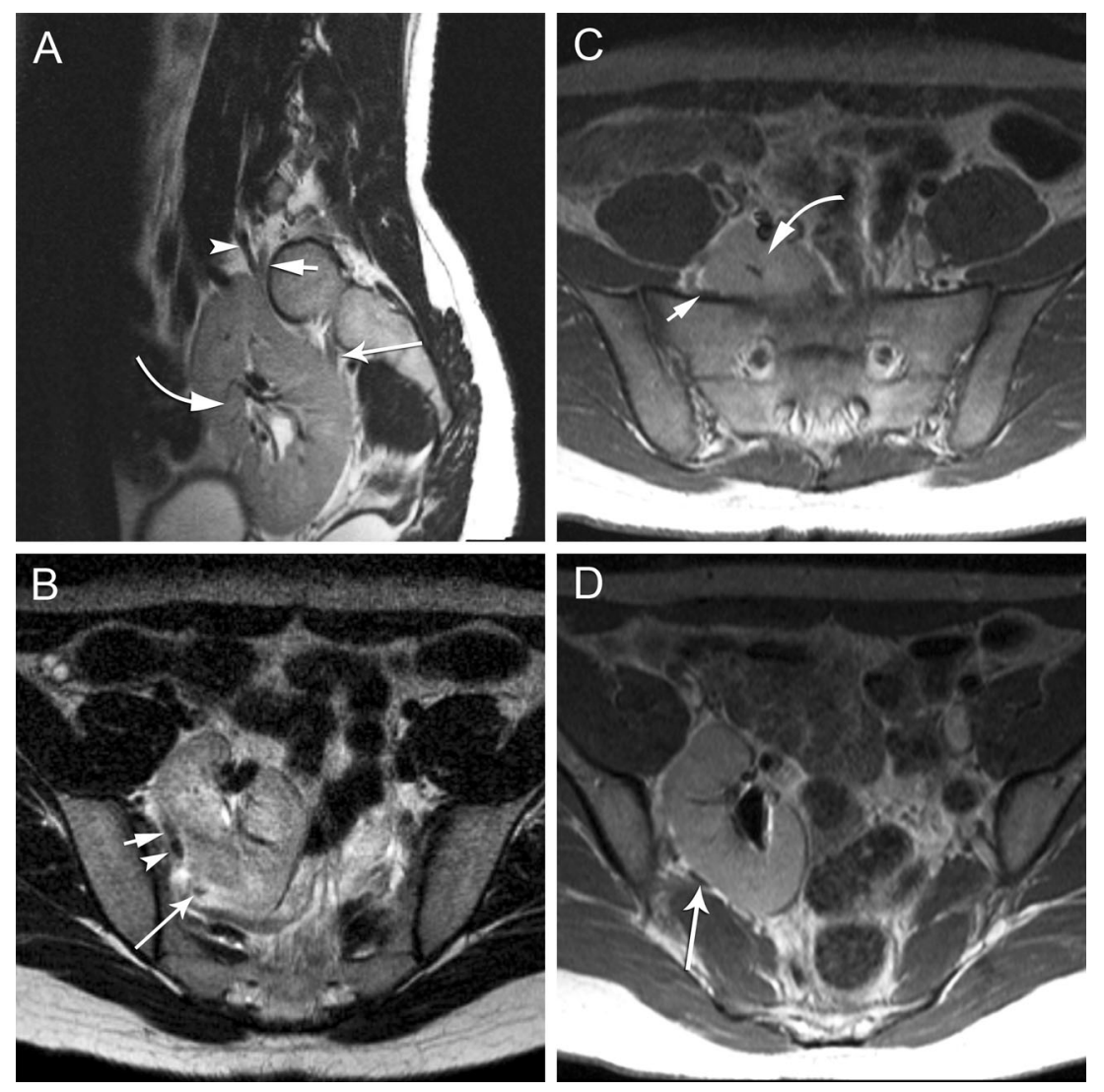
narrow space in the true pelvis and by changes in patient position. Whether changes in renal collecting system volume would affect the degree of compression is not known, but possible. The patient's symptoms though did change with a cross-legged position or prolonged standing.

A limitation of our report is that the patient did not undergo surgery, and postsurgical confirmation of relief of symptoms is not available, but considering the relatively minor neurological symptoms, response to medical therapy, and the need for a complex surgery, the patient did not undergo surgery. The available electrodiagnostic studies were unremarkable, but these tests may be unrevealing in diagnosis of some neuropathies, particularly, more proximal and inaccessible neuropathies [8,9].

In conclusion, given the history, physical exam findings, and morphological findings on imaging, it was surmised that as the consequence of the very rare position of this patient's ectopic kidney, sciatic compressive mononeuropathy had developed. Physicians should consider other rare causes of mechanical lumbosacral plexus compression in patients with sciatic symptomatology.

\section{References}

1. Cinman NM, Okeke ZSA (2007) Pelvic kidney: associated diseases and treatment. J Endourol 21:836-842

2. Li Q-S, Huo W-Q, Nie Z-L, Wang H-F, Liang P-H, Jin F-S (2010) Acute femoral neuropathy following renal transplantation: a retrospective, multicenter study in China. Transplant Proc 42:1699-1703. doi: 10.1016/j.transproceed.2010.02.082

3. Donnelly PK, Ponsford S (1995) Acute sciatic neuropathy complicating living donor renal transplantation. Aust N Z J Surg 65:290-291. doi:10.1111/j.1445-2197.1995.tb00633.x

4. Sheih C-P, Liu M-B, Hung C-S, Yang K-H, Chen W-Y, Lin C-Y (1989) Renal abnormalities in schoolchildren. Pediatrics 84:1086-1090

5. La L (1996) Rare case of a kidney situated in the lesser pelvis. The diagnostic difficulties. Akush Ginekol (Sofiia) 35:40-41

6. Sharma KR, Cross J, Santiago F, Ayyar DR, Burke G (2002) Incidence of acute femoral neuropathy following renal transplantation. Arch Neurol 59:541-545

7. Nikoobakht M, Mahboobi A, Saraji A, Mehrsai A, Emamzadeh A, Mahmoudi MT, Pourmand G (2007) Pelvic nerve neuropathy after kidney transplantation. Transplant Proc 39:1108-1110. doi:10.1016/j. transproceed.2007.03.085

8. Barr K (2013) Electrodiagnosis of lumbar radiculopathy. Phys Med Rehabil Clin N Am 24:79-91. doi:10.1016/j.pmr.2012.08.011

9. Landau ME, Campbell WW (2013) Clinical features and electrodiagnosis of ulnar neuropathies. Phys Med Rehabil Clin N Am 24:49-66. doi:10.1016/j.pmr.2012.08.019 